\title{
Effect of siRNA-mediated downregulation of VEGF in Tca8113 cells on the activity of monocyte-derived dendritic cells
}

\author{
YAN-HONG NI ${ }^{1,2^{*}}$, ZHI-YONG WANG ${ }^{2 *}$, XIAO-FENG HUANG ${ }^{2 *}$, PEI-HUA SHI ${ }^{2}$, \\ WEI HAN $^{1,2}$, YA-YI HOU ${ }^{3}$, ZI-CHUN HUA ${ }^{4}$ and QIN-GANG HU ${ }^{1,2}$ \\ ${ }^{1}$ Central Laboratory of Stomatology, Nanjing Stomatological Hospital, Institute and Hospital of Nanjing Medical School, \\ Nanjing University, Nanjing 210008; ${ }^{2}$ Department of Oral and Maxillofacial Surgery, Institute and Hospital of \\ Nanjing Medical School, Nanjing University, Nanjing 210008; ${ }^{3}$ Immunology Laboratory, Medical School \\ and State Key Laboratory of Pharmaceutical Biotechnology, Nanjing University, Nanjing 210093; \\ ${ }^{4}$ The State Key Laboratory of Pharmaceutical Biotechnology, Nanjing 210093, P.R. China
}

Received November 1, 2011; Accepted December 28, 2011

DOI: $10.3892 / \mathrm{ol} .2012 .568$

\begin{abstract}
Vascular endothelial growth factor (VEGF) is a tumor angiogenesis factor that is important in immune regulation. In our previous study, we found that VEGF expression in the peripheral blood and neoplasm nest from patients with oral squamous cell carcinoma (OSCC) was positively correlated with the course of disease, while an inverse correlation between VEGF expression and dendritic cells (DCs) was identified in the peripheral blood. Therefore, in the present study, we investigated whether inhibition of human VEGF in the human tongue carcinoma cell line Tca8113 had effects on the activity of monocyte-derived DCs. We knocked down the expression of human VEGF in Tca8113 cells using the small interfering RNA (siRNA) technique. Tca8113 cells pre-transfected with siRNA targeting VEGF were co-cultured with monocyte-derived immature and mature DCs. Cell proliferation was evaluated by a WST-8 assay. Cell apoptosis, cell cycle and cell phenotypes were determined by flow cytometry. The data revealed that downregulation of the human VEGF significantly inhibited the proliferation of Tca8113 cells and increased apoptosis. Inhibition of human VEGF arrested the cell cycle of Tca8113 cells at the G0/G1 phase. Our results showed that the co-culture
\end{abstract}

Correspondence to: Dr Qin-Gang Hu, Department of Oral and Maxillofacial Surgery, School of Stomatology, Nanjing University Medical Center, 30 Zhong Yang Road, Nanjing 210008, P.R. China E-mail: qinganghu@hotmail.com

*Contributed equally

Abbreviations: APC, antigen presenting cell; DC, dendritic cell; GM-CSF, granulocyte macrophage colony-stimulating factor; IL-4, interleukin-4; OSCC, oral squamous cell carcinoma; PBMC, peripheral blood mononuclear cell; PE, phycoerythrin; TNF- $\alpha$, tumor necrosis factor $\alpha$; VEGF, vascular endothelial growth factor

Key words: oral squamous cell carcinoma, vascular endothelial growth factor, RNA interference, dendritic cells of DCs with Tca8113 cells markedly inhibited the expression of the mature markers of DCs including HLA-DR, CD80, CD86, CD40 and CD1a, as well as the immature marker CD83, while inhibition of human VEGF in Tca8113 cells significantly reversed these effects. Therefore, human VEGF in Tca8113 cells may not only regulate the cell proliferation and apoptosis of oral squamous cell carcinoma cells, but may also inhibit DC maturation.

\section{Introduction}

Head and neck squamous cell carcinomas (HNSCCs) are common malignancies. Combinations of chemotherapy, surgery and radiotherapy have shown beneficial effects on the local and regional control of these diseases. However, these strategies have been unsuccessful in the treatment of local recurrence and metastatic diseases $(1,2)$. Therefore, a number of investigators are examining new methods to overcome the shortcomings of these treatments.

Various types of cancer express vascular endothelial growth factor (VEGF), which induces neoangiogenesis and blockade of angiogenesis, a critical step in the development and metastasis of solid tumors, and has become a major useful approach in cancer therapy (3). Our previous studies revealed that VEGF expression in the peripheral blood and neoplasm nest of patients with oral squamous cell carcinoma (OSCC) was positively correlated with the course of this disease and was inversely correlated with dendritic cells (DCs) in the peripheral blood (4). DCs are specialized antigen presenting cells, which acquire, process and present antigens, such as tumor-associated antigens, to T cells for the induction of antigen-specific immune responses. Immature DCs are known to migrate into tumor beds and capture tumor-derived antigens for presentation to specific T cells. However, adaptive immune responses are often hampered in cancer patients (5). Panayiotis et al reported that VEGF was associated with worse overall survival in patients with HNSCC (6). We found that a low density of mature DC infiltrated into tumor tissue, which may be caused by the immunosuppressive microenvironment of OSCC (4). 
Considering that the blockade of VEGF in a mouse model leads to increased antigen uptake and migration of tumorassociated DCs (7), we speculated that inhibition of human VEGF increases the differentiation and maturation of DCs in OSCC, resulting in an increased inhibition of tumorigenesis. In the present study, we investigated whether inhibition of human VEGF in the human tongue carcinoma cell line Tca8113 had an impact on the activity of monocyte-derived DCs. We downregulated the expression of human VEGF in Tca8113 cells using the small interfering RNA (siRNA) technique. We analyzed the expression of mature markers on DCs following the co-culture of DCs with VEGF-downregulated Tca8113 cells.

\section{Materials and methods}

Cell line. Tca8113 cells were kindly provided by the Ninth People's Hospital of Shanghai, China. The cells were cultured in RPMI-1640 medium (Gibco, Grand Island, NY, USA) supplemented with $10 \%$ heat-inactivated fetal bovine serum (FBS, HyClone Corp, USA). The cultures were incubated at $37^{\circ} \mathrm{C}$ in an atmosphere of $5 \%$ humidified $\mathrm{CO}_{2}$.

RNA extraction and reverse transcription polymerase chain reaction $(R T-P C R)$. Total RNA was isolated using the TRIzol ${ }^{\circledR}$ reagent (Invitrogen, USA) according to the manufacturer's instructions. For RT-PCR, the following DNA primers were synthesized by Invitrogen: Human VEGF, sense primer 5'-GAGGGCAGAATCATCACGAAGT-3' and anti-sense primer 5'-GGTGAGGTTTGATCCGCATAA-3'; human GAPDH, sense primer 5'-TGGTCTCCTCTGACTTCAAC-3' and anti-sense primer 5'-GTGAGGGTCTCTCTCTTCCT-3'. Human GAPDH was used as an internal control. The PCR program included pre-incubation for $3 \mathrm{~min}$ at $94^{\circ} \mathrm{C}$, followed by 28 cycles of $30 \mathrm{sec}$ at $94^{\circ} \mathrm{C}, 30 \mathrm{sec}$ at $55^{\circ} \mathrm{C}, 30 \mathrm{sec}$ at $72^{\circ} \mathrm{C}$, and final extension for $10 \mathrm{~min}$ at $72^{\circ} \mathrm{C}$. Amplification was performed using the Applied Biosystems Veriti (ABI Corporation, USA). The PCR products were analyzed on an agarose gel and photographed on a UV-transilluminator.

Protein expression analysis. Total protein expression was analyzed with western blotting as previously described (8). Briefly, the cells were solubilized in lysis buffer $[10 \mathrm{mM}$ Tris-HCl (pH 7.5), 150 mM NaCl, 1\% NP-40, 1 mM PMSF, and $10 \mathrm{ng} / \mathrm{ml}$ soybean trypsin inhibitor]. The proteins were transferred onto a polyvinylidene difluoride (PVDF) filter membrane (Amersham Biosciences). Following blocking with 5\% non-fat dry milk in PBS containing $0.01 \%$ Tween-20 (T-PBS), the membrane was blotted with the anti-VEGF $(1: 200$; eBioscience Inc.) or GAPDH (1:1000; Abcam Inc.) polyclonal antibodies. The blots were washed in T-PBS for $15 \mathrm{~min}$ each and then developed using peroxidase-labeled rabbit anti-mouse $\operatorname{IgG}(1: 2000)$ for $60 \mathrm{~min}$ at room temperature. The protein was visualized by enhanced chemiluminescence.

Construction of siRNA expression vectors. The mammalian cell expression vector pRNATU6.1/Neo, carrying a green fluorescent protein (GFP) reporter gene, was purchased from GenScript Corp. (USA) and was used to generate biologically active siRNAs under the U6 promoter. Four 21-nucleotide sequences targeting VEGF were designed and cloned into pRNATU6.1/Neo generating VEGFsiRNA-V1, VEGFsiRNA-V2, VEGFsiRNA-V3 and VEGFsiRNA-V4. A siRNA sequence that is not homologous to any known human DNA sequence was used as a negative control. The constructs were verified by DNA sequencing (Bioasia, China). The effects of RNA interference on the constructs were investigated by RT-PCR.

Cell transfection. A total of $1.0 \times 10^{4} \mathrm{Tca} 8113$ cells were plated in each well of a 12-well plate (Costar, Corning, USA). Upon attaining 90-95\% confluence, the cells were transfected with $2 \mu \mathrm{gDNA}$ and $4 \mu \mathrm{lLipofectamine} 2000$ (Invitrogen,USA)perwell according to the manufacturer's instructions. G418 $(400 \mathrm{ng} / \mathrm{ml})$ (Life Technologies) was added to the medium after $48 \mathrm{~h}$ of culture, and the cells were cultured for two weeks to permit selection.

Cell viability analysis. Cell viability was assessed with a cell counting kit according to the manufacturer's instructions (Dojin Laboratories, Kumamoto, Japan). Briefly, cells at a density of $3 \times 10^{3}$ cells/well were plated in a 96-well plate. Following 5 days of culture, $10 \mu 1$ 4-[3-(2-methoxy-4-nitropheny[T1]1)-2-(4nitrophenyl)-2H-5-tetrazolio]-1, 3-benzene disulfonate sodium salt (WST-8) was added to each well. Following incubation for $1,2,3$ and $4 \mathrm{~h}$, the absorbance was measured at $450 \mathrm{~nm}$ using a multi-detection microplate reader (Hynergy ${ }^{\mathrm{TM}}$ HT, Bio-Tek).

Cell cycle and apoptosis analysis. The cell cycle was analyzed with propidium iodide (PI) as previously described (9). Briefly, the cells were fixed with $70 \%$ ethanol and were subsequently treated with $0.25 \mathrm{mg} / \mathrm{ml}$ RNase. Finally, cells were stained with $20 \mu \mathrm{g} / \mathrm{ml}$ propidium iodide (PI). Annexin V/PI was used for the apoptosis analysis. Briefly, cells were stained with Annexin V-APC (Bender MedSystems Inc., USA) for $15 \mathrm{~min}$ and then incubated with PI for an additional $10 \mathrm{~min}$. Viable cells were $\mathrm{PI}^{-}$Annexin $\mathrm{V}^{-} \mathrm{APC}$ and non-viable cells, which underwent necrosis, were $\mathrm{PI}^{+} \mathrm{Annexin} \mathrm{V}^{-} \mathrm{APC}-$. Early apoptotic cells were $\mathrm{PI}^{-} \mathrm{Annexin} \mathrm{V}^{-} \mathrm{APC}^{+}$, whereas late apoptotic dead cells exhibited $\mathrm{PI}^{+} \mathrm{Ann}$ exin $\mathrm{V}^{-} \mathrm{APC}^{+}$.

Generation of monocyte-derived DCs. Peripheral blood mononuclear cells (PBMCs) were isolated from human blood using Ficoll/Hypaque (density 1.077 g/ml, Shanghai Shisheng Cytobiotechnological Corp., China) according to the manufacturer's instructions. Following the last wash, PBMCs were resuspended in RPMI-1640 with 10\% FBS and were then plated in a 24-well culture plate. Following $3 \mathrm{~h}$ of incubation, monocytes were purified by removing the non-adherent cells in the supernatant. To obtain immature DCs, monocytes were cultured with $100 \mathrm{ng} / \mathrm{ml} \mathrm{rhGM}-\mathrm{CSF}$ and $40 \mathrm{ng} / \mathrm{ml} \mathrm{rhIL}-4$ (Peprotech UK) for five days. Mature DCs were obtained following an additional two days of culture in medium supplemented with $100 \mathrm{ng} / \mathrm{ml} \mathrm{rhGM}-\mathrm{CSF}, 40 \mathrm{ng} / \mathrm{ml} \mathrm{rhIL}-4$ and $20 \mathrm{ng} / \mathrm{ml}$ rhTNF- $\alpha$ (Peprotech, UK).

Cell co-culture. Cell-to-cell contacting co-culture experiments were performed as previously described (ref.). In one set of experiments, immature DCs were co-cultured with Tca8113 or VEGFsiRNA-treated Tca8113 cells. In another set 
A
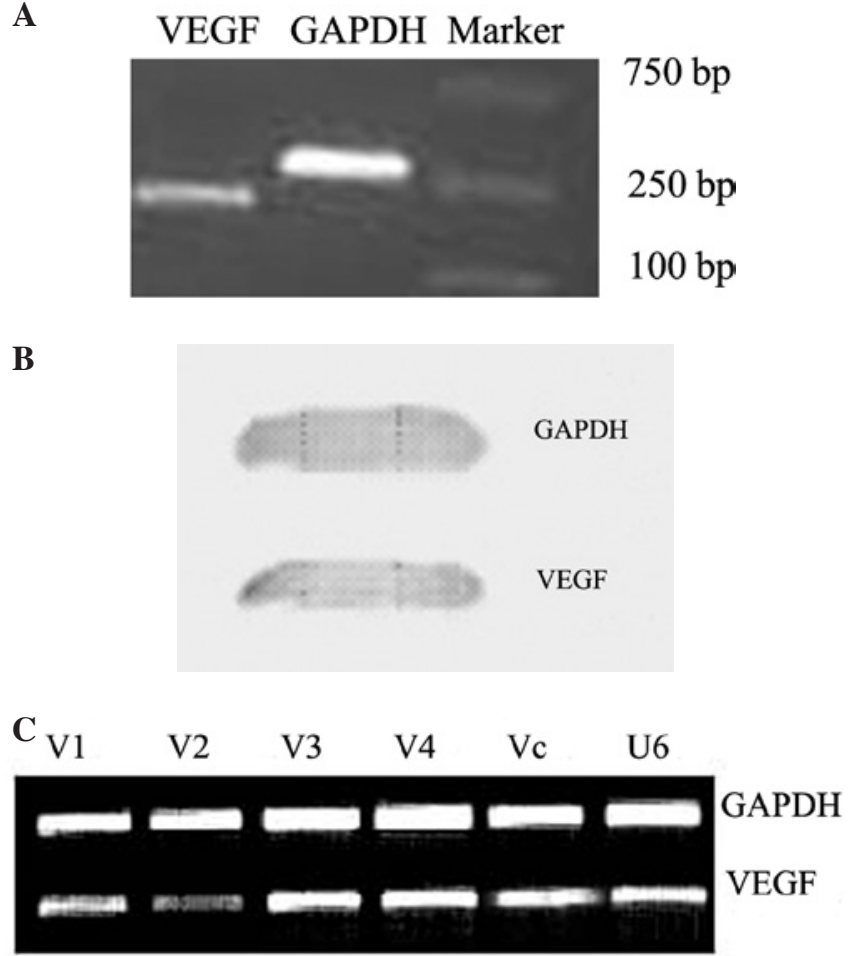

Figure 1. The expression of VEGF in the Tca8113 cell line and selection of the effective VEGF siRNA sequence in Tca8113 cells. The expression of VEGF in Tca8113 was detected by (A) RT-PCR and (B) by western blotting. (C) The efficiency of transfection reached approximately $90 \%$ as observed by fluorescence microscopy. (D) VEGF siRNA-V2 effectively inhibited VEGF expression in Tca8113 cells after $48 \mathrm{~h}$ by RT-PCR. V1: VEGFsiRNA-V1. V2: VEGFsiRNA-V2. V3: VEGFsiRNA-V3. V4: VEGFsiRNA-V4. Vc: siRNA-Vc. U6: empty vector. VEGF, vascular endothelial growth factor; siRNA, small interfering RNA; RT-PCR, reverse transcription polymerase chain reaction.

of experiments, mature DCs were co-cultured with Tca8113 or VEGFsiRNA-treated Tca8113 cells. The co-culturing conditions were the same for both sets of experiments where RPMI-1640 was supplemented with $10 \% \mathrm{FBS}, 100 \mathrm{ng} / \mathrm{ml}$, rhGM-CSF, $40 \mathrm{ng} / \mathrm{ml} \mathrm{rhIL}-4$ and $20 \mathrm{ng} / \mathrm{ml} \mathrm{rhTNF}-\alpha$. The ratio of DC to tumor cells was $1: 1$ at $1 \times 10^{5}$ cells $/ \mathrm{ml}$.

Analysis of the DC phenotype with flow cytometry. The DC phenotype was analyzed with flow cytometry. Mouse anti-HLADR-Percp-cy5.5, CD80 (PE), CD83 (PE), CD1a (PE) and CD14 (APC), as well as corresponding isotypes were used, and murine isotype APC-conjugated, PE-conjugated and Percp-cy5.5 IgG antibodies were purchased from eBioscience (USA). The antibodies for CD86 (APC) and CD40 (APC) were from Caltag (USA), while the murine isotype served as a negative control.

Statistical analysis. The data were analyzed using the SPSS version 13.0 software. Differences between paired groups were analyzed using Student's paired t-test. $\mathrm{P}<0.05$ was considered to indicate a statistically significant difference.

\section{Results}

Downregulation of VEGF in Tca8113 cells. The PCR and western blotting results revealed that Tca8113 cells highly
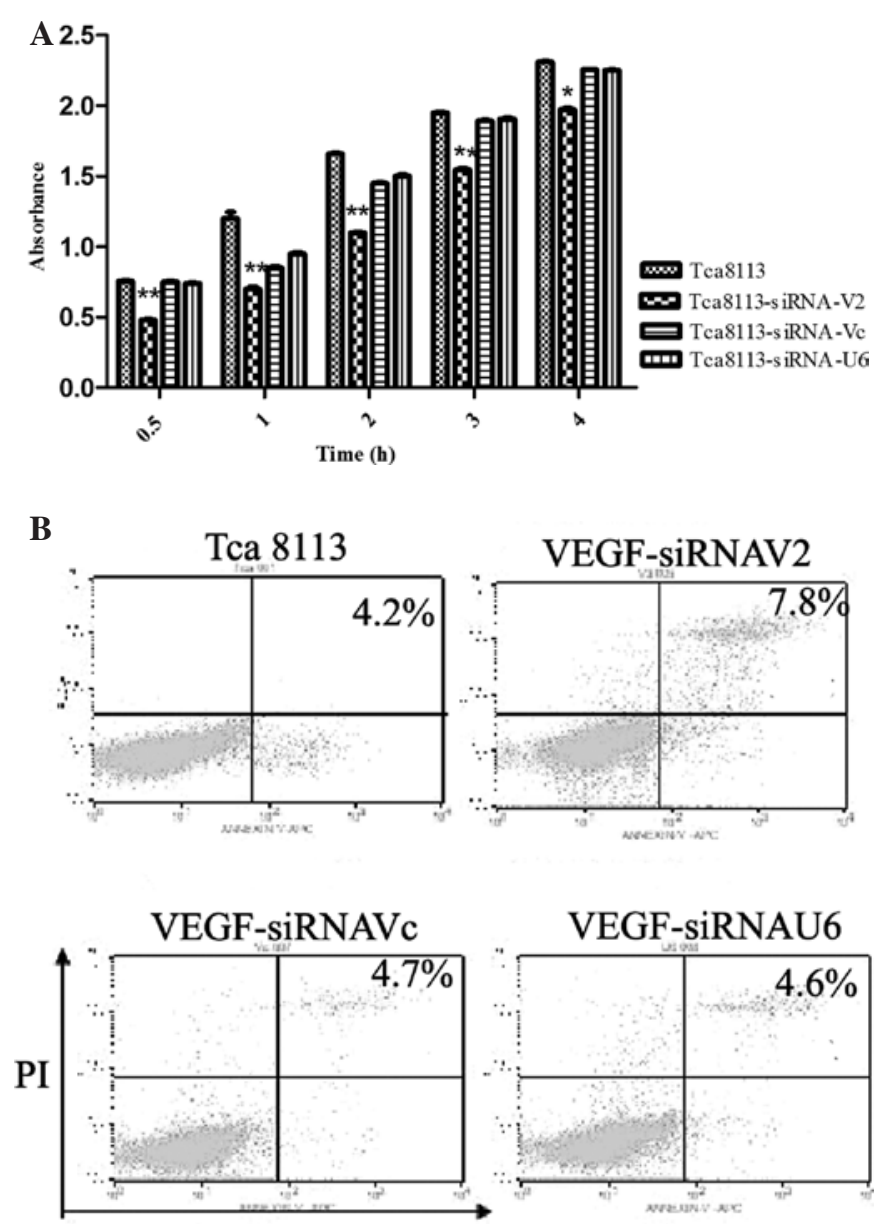

Annexin V

\section{C}

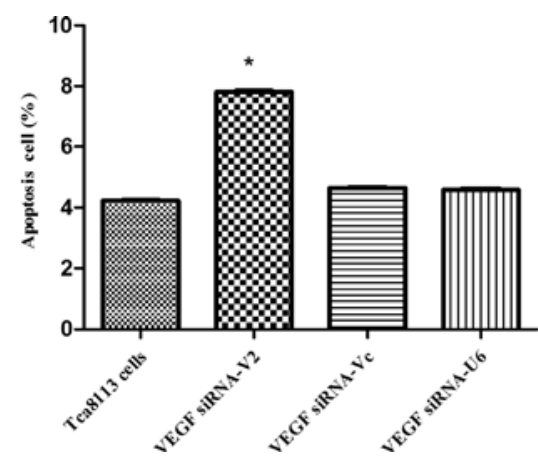

Figure 2. Effects of VEGF siRNA-V2 on the viability and apoptotic state of Tca8113 cells. (A) VEGF siRNA-V2 inhibited Tca8113 cell viability as assayed with a cell counting kit using WST- 8 dye. Results are shown as the mean absorbance \pm SEM of three independent experiments. (B) VEGF siRNA-V2 promoted Tca8113 cell apoptosis. The cells were detected by Annexin V/PI double staining, and representative dot plots of Annexin-V/ PI staining are shown: lower left quadrant, viable (double negative) population; lower right quadrant, apoptotic $\left(\mathrm{AnnexinV}^{+} / \mathrm{PI}\right)$ population; upper left quadrant, necrosis (Annexin $\mathrm{V}^{-} / \mathrm{PI}^{+}$) population; upper right quadrant, late apoptotic dead cells (Annexin $\mathrm{V}^{+} / \mathrm{PI}^{+}$). (C) The percentages of Annexin V positive cells are shown. Data are shown as the means \pm SEM of three independent experiments. ${ }^{*} \mathrm{P}<0.05$ versus control. VEGF, vascular endothelial growth factor; siRNA, small interfering RNA; PI, propidium iodide.

expressed VEGF at the mRNA and protein levels (Fig. 1A and B). We blocked the expression of VEGF in Tca8113 cells by transfection with VEGFsiRNA-V1, VEGFsiRNA-V2, VEGFsiRNA-V3 and VEGFsiRNA-V4, respectively. The transfection efficiency reached approximately $90 \%$. Following 
A
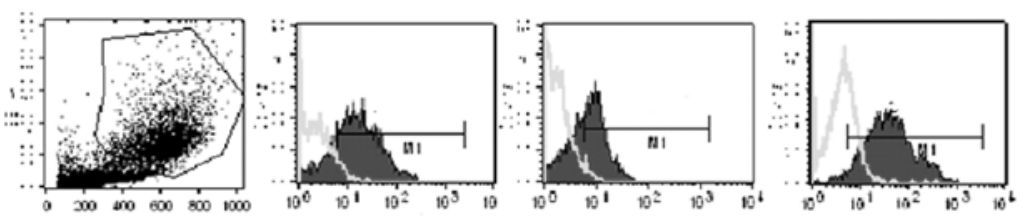

HLA-DR

CD80
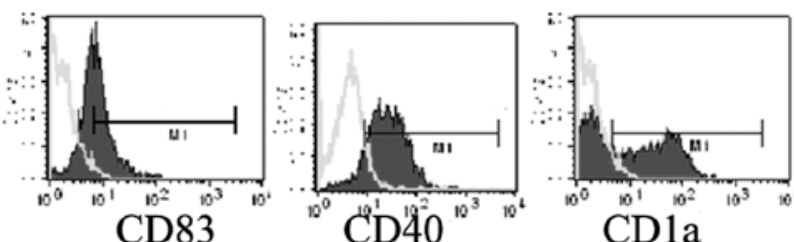

CD86

B
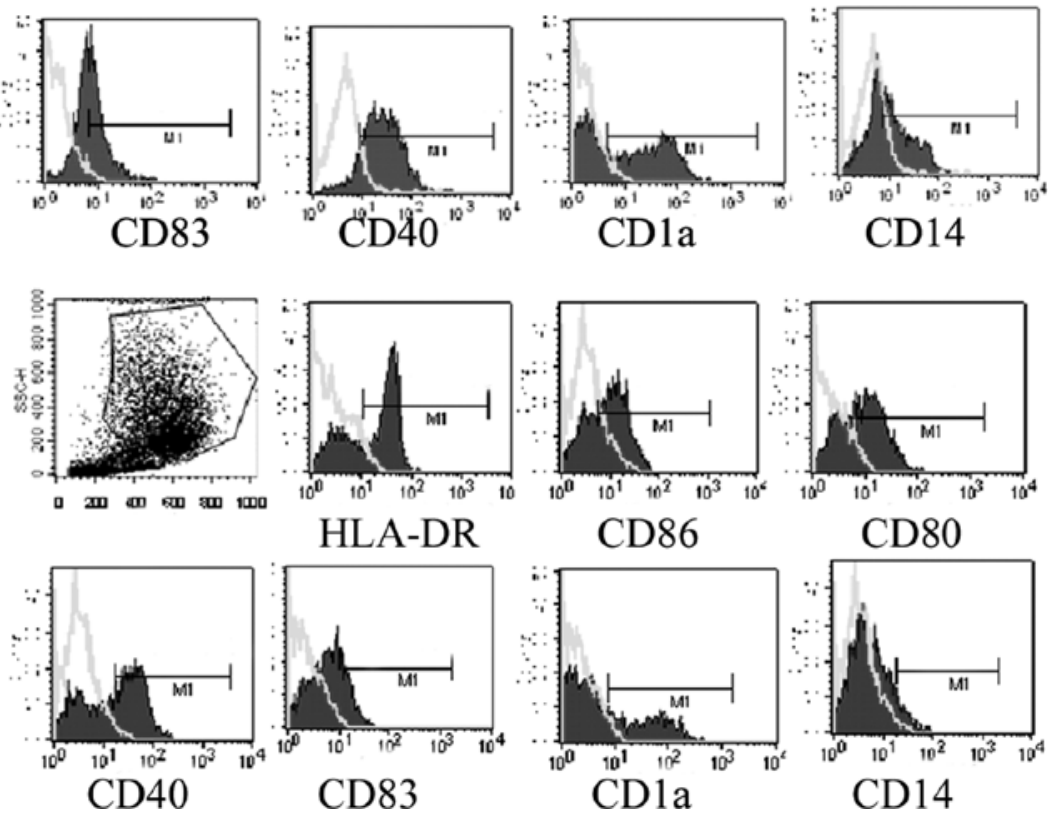

CD80

C

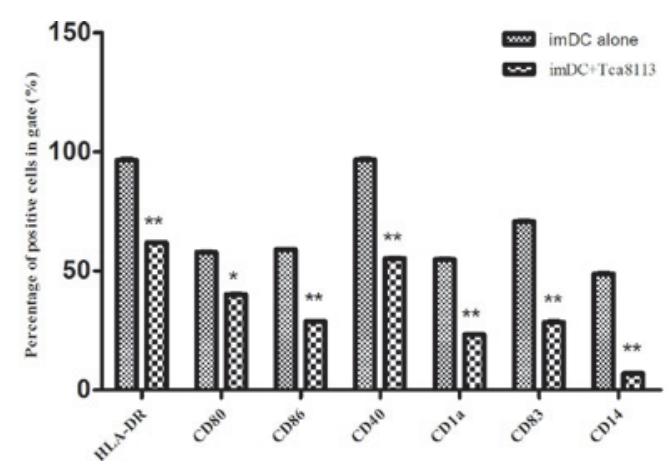

D
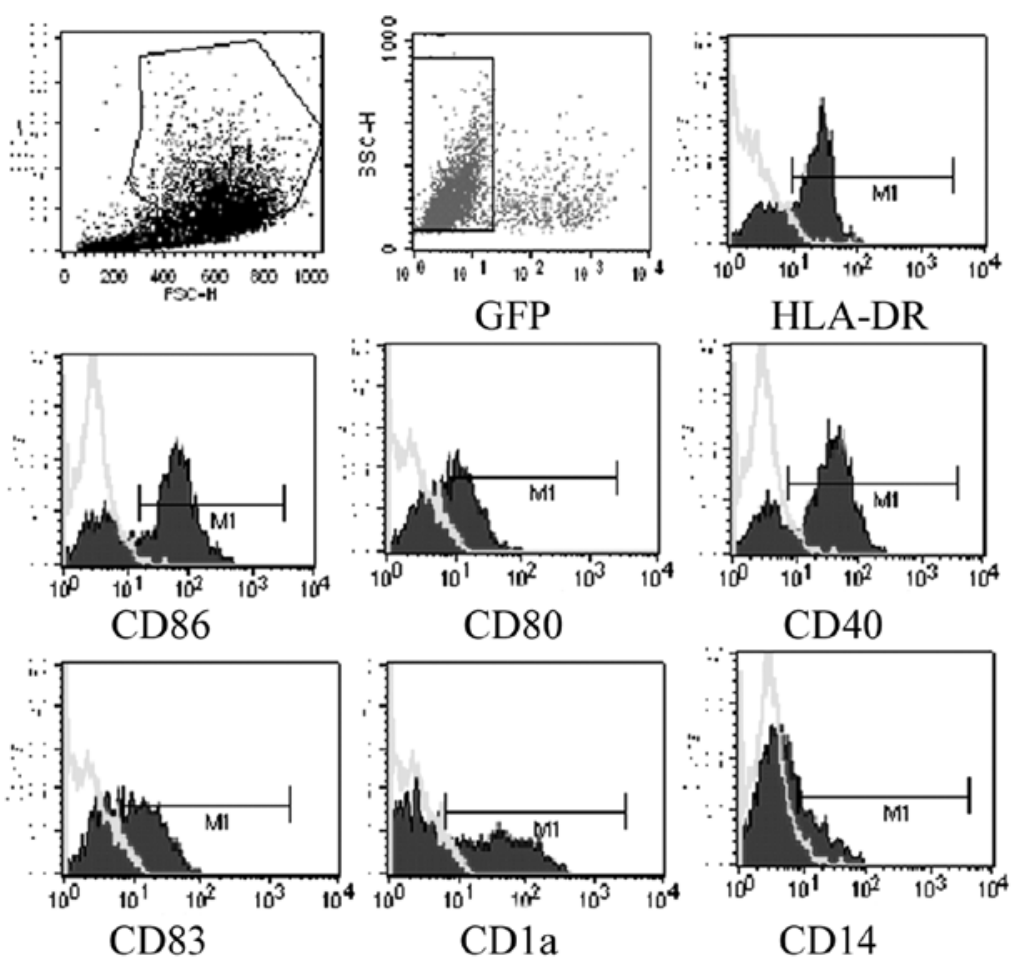
$\mathbf{E}$

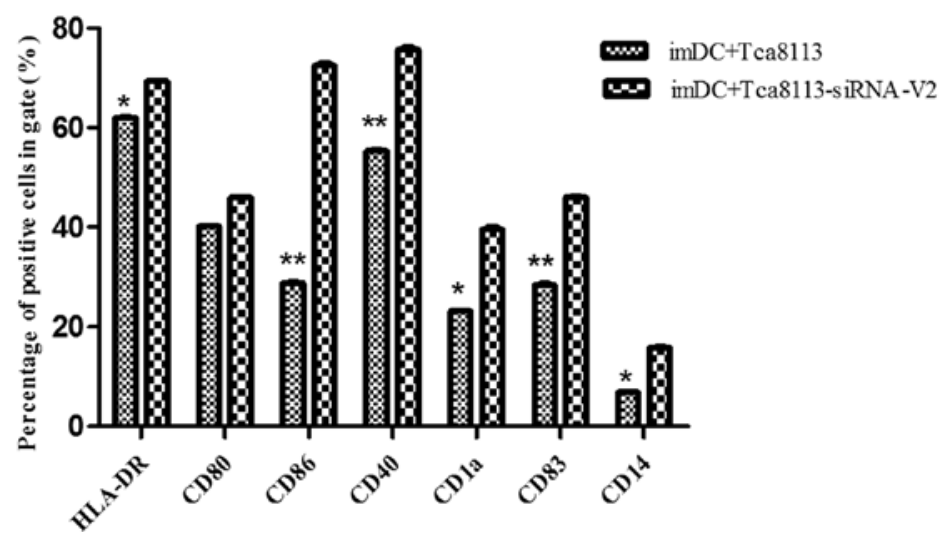

Figure 3. VEGF suppressed the differentiation of immature DCs into mature DCs. Immature DCs were cultured alone or with Tca8113 cells in medium containing GM-CSF, IL-4 and TNF- $\alpha$ for 48 h. (A) The expression of HLA-DR, CD40, CD80, CD83 and CD1a were analyzed by flow cytometry. (B) The expression of HLA-DR, CD40, CD80, CD83 and CD1a decreased when immature DCs were co-cultured with Tca8113 cells for $48 \mathrm{~h}$. (C) The expression of HLA-DR, CD40, CD80, CD83 and CD1a was suppressed in DCs when co-cultured with Tca8113 cells. (D) The expression of HLA-DR, CD40, CD80, CD83 and CD1a increased when immature DCs were co-cultured with VEGFsiRNA-V2-treated Tca8113 cells for $48 \mathrm{~h}$. (E) The expression of HLA-DR, CD40, CD80, CD83 and CD1a was higher on immature DCs when co-cultured with VEGF siRNA-V2-treated Tca8113 cells than with wild-type Tca8113 cells. Data are the mean \pm SD of triplicate samples. "P $<0.05$ versus control. DCs, dendritic cells; VEGF, vascular endothelial growth factor; siRNA, small interfering RNA; GM-CSF, granulocyte macrophage colony-stimulating factor; IL-4, interleukin-4; TNF- $\alpha$, tumor necrosis factor $\alpha$.

Table I. Cell cycle states of Tca8113 cells with or without transfection with siRNA plasmid (\%).

\begin{tabular}{lccc}
\hline Groups & G0-G1 & G2-M & S \\
\hline Tca8113 & $57.00 \pm 0.98$ & $8.10 \pm 1.22$ & $34.90 \pm 0.85$ \\
Tca8113-shRNA-V2 & $63.06 \pm 0.76$ & $4.95 \pm 1.30$ & $31.99 \pm 0.34$ \\
Tca8113-shRNA-VC & $58.44 \pm 1.38$ & $7.58 \pm 0.56$ & $33.98 \pm 0.67$ \\
Tca8113-shRNA-U6 & $58.76 \pm 0.51$ & $6.79 \pm 1.36$ & $34.45 \pm 0.67$
\end{tabular}

G418 selection for two weeks, RT-PCR analysis revealed that VEGF was significantly downregulated in Tca 8113 cells transfected with VEGFsiRNA-V2, as compared with the controls (Fig. 1C). Therefore, we selected VEGFsiRNA-V2 as the most effective plasmid for use in subsequent experiments.

Downregulation of VEGF in Tca8113 cells inhibited cell proliferation and cell cycle and induced apoptosis. To determine whether interference of VEGF regulated cell growth, cell viability was assessed with a cell counting kit as described in Materials and methods. Downregulation of VEGF in Tca8113 cells by transfection with VEGF siRNA V2 significantly inhibited cell proliferation as compared to the negative controls (Fig. 2A). In contrast to the controls, the cell cycle of Tca8113 cells transfected with VEGFsiRNA-V2 was significantly arrested at the G0/G1 phase (Table I).

To examine whether suppression of VEGF expression induced apoptosis, the cells were stained with Annexin V and PI. The data revealed that apoptotic cells increased significantly in Tca8113 cells following downregulation of VEGF (Fig. 2B and C). These results indicated that VEGF inhibited cell growth and induced apoptosis of Tca8113 cells.

$V E G F$ inhibited the differentiation of immature DCs into mature DCs. To analyze whether downregulation of VEGF in
Tca8113 cells had an impact on the differentiation of immature DCs into mature DCs, we co-cultured the immature DCs with Tca8113 cells or VEGF-downregulated Tca8113 cells in the presence of GM-CSF, IL- 4 and TNF- $\alpha$. The mature markers of DCs including HLA-DR, CD80, CD86, CD40 and CD1a, as well as the immature marker $\mathrm{CD} 83$, were analyzed by flow cytometry (Fig. 3A). The expression of HLA-DR, CD80, CD86, CD40, CD83, CD1a and CD14 on these DCs was markedly decreased by Tca8113 cells (Fig. 3B and 3C). However, the downregulation of VEGF in Tca8113 cells significantly reversed these effects (Fig. 3D and E). This suggested that VEGF released by Tca8113 had an inhibitory role on the differentiation of immature DCs into mature DCs.

VEGF-downregulated Tca8113 cells increased the proportion of mature DCs. To determine whether the downregulation of VEGF in Tca8113 cells regulated the activity of mature DCs, we co-cultured mature DCs with Tca 8113 cells or VEGFsiRNA-treated Tca8113 cells. The mature markers of DCs including HLA-DR, CD80, CD86, CD40 and CD1a, as well as the immature marker CD83, were assessed by flow cytometry (Fig. 4A). In contrast to the controls, the expression of HLA-DR, CD80, CD86, CD40 and CD14 on mature DCs following co-culture with Tca8113 cells was markedly decreased, while the immature marker CD83 was increased (Fig. 4B and C). Downregulation of VEGF in Tca8113 cells significantly reversed these effects, whereas the expression of HLA-DR, CD86, CD80, CD40 and CD14 on mature DCs increased (Fig. 4D and 4E). This difference indicated that Tca8113 cells released VEGF, and may regulate the activity of mature DCs.

\section{Discussion}

It is well known that human VEGF is highly expressed in numerous types of cancer. VEGF is particularly upregulated 
A
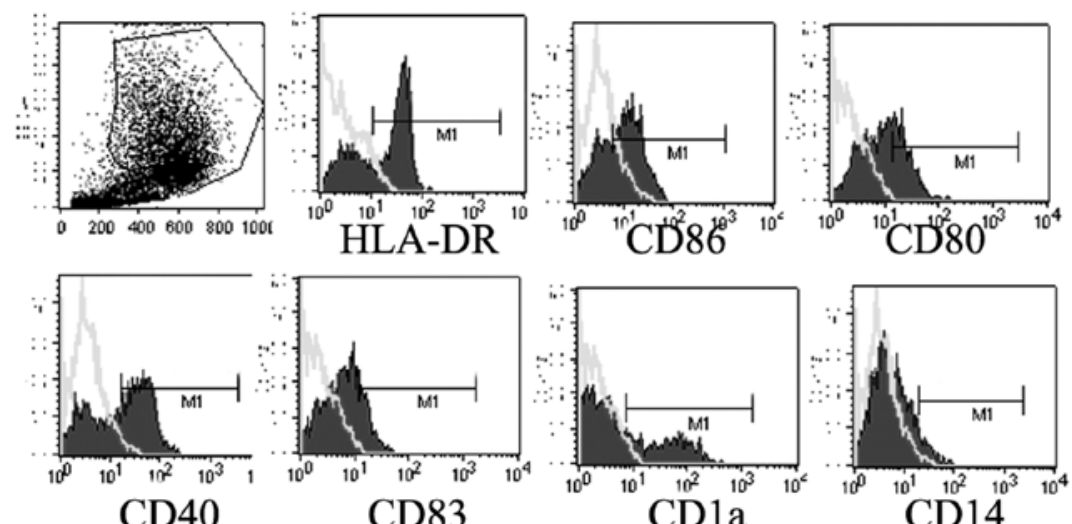

B
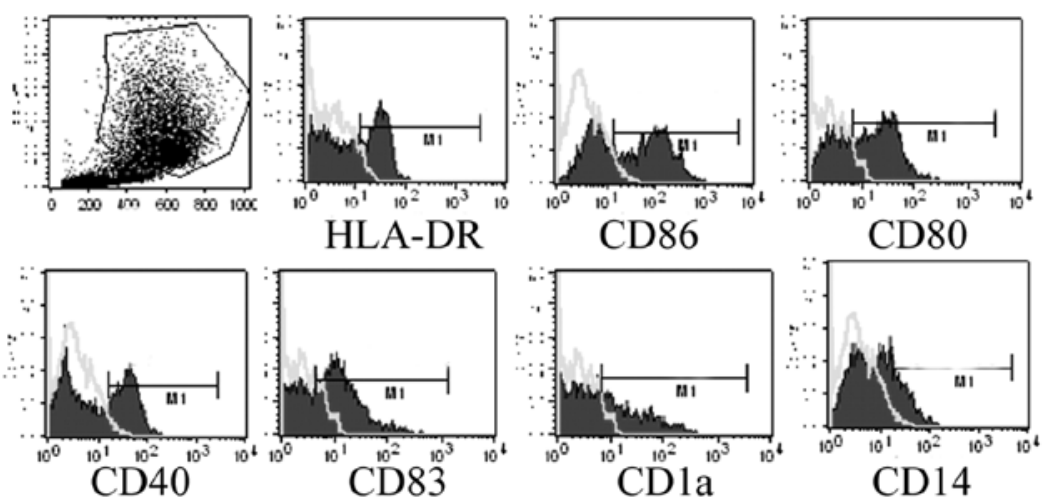

C

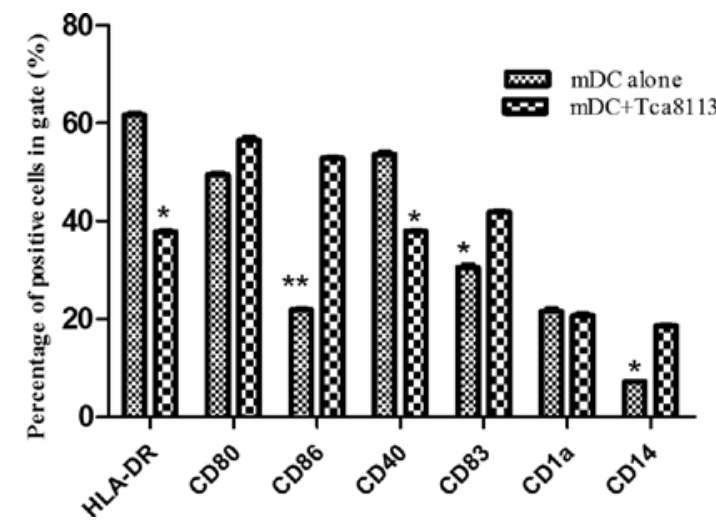

D
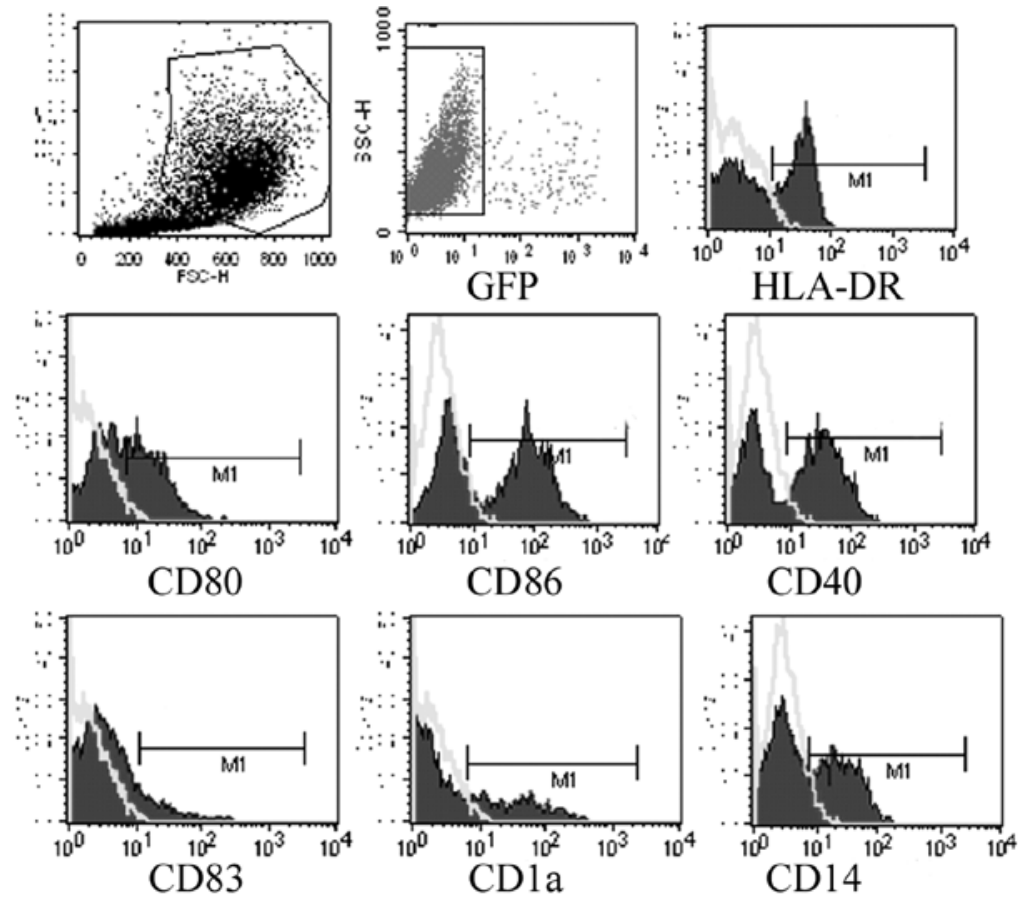
E

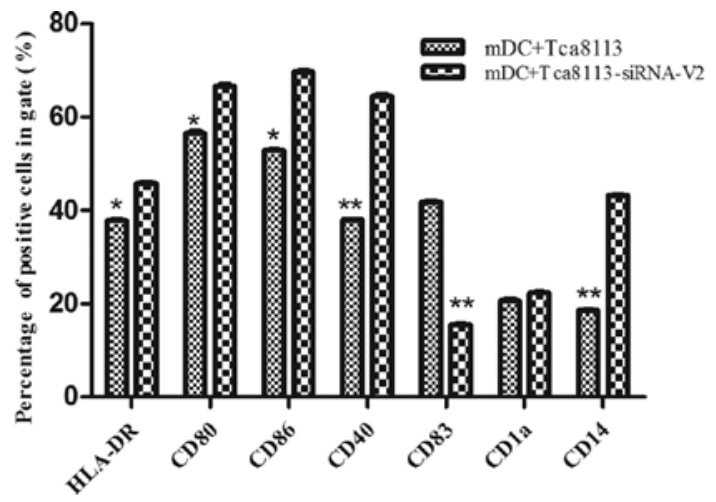

Figure 4. VEGF also suppressed the surface molecules of mature DCs. The mature DCs were cultured alone or co-cultured with Tca8113 cells in the RPMI-1640 medium containing 10\% FBS for $48 \mathrm{~h}$. We analyzed the DC surface molecules excluding the Tca8113 cells by setting up two gates. (A) The expression of HLA-DR, CD40, CD80, CD83 and CD1a increased when mature DCs were cultured alone for 48 h. (B) The expression of HLA-DR, CD40, CD80, CD83 and CD1a was decreased when mature DCs were co-cultured with Tca8113 cells for $48 \mathrm{~h}$. (C) The expression of HLA-DR, CD40, CD80, CD83 and CD1a was suppressed in mature DC when co-cultured with Tca8113 cells. (D) The expression of HLA-DR, CD40, CD80, CD83 and CD1a was increased when mature DCs were co-cultured with VEGF siRNA-V2-treated Tca8113 cells for $48 \mathrm{~h}$. (E) The expression of HLA-DR, CD40, CD80, CD83 and CD1a was higher on mature DCs when co-cultured with VEGF siRNA-V2-treated Tca8113 cells than with wild-type Tca8113 cells. Data represent the mean \pm SD of triplicate samples. ${ }^{*}<0.05$ versus control. DCs, dendritic cells; FBS, fetal bovine serum; VEGF, vascular endothelial growth factor; siRNA, small interfering RNA.

in response to hypoxia, activated oncogenes or inactivated tumor suppression genes (10). Therefore, suppression of VEGF expression at the RNA or protein levels may inhibit tumor growth. It is reported that VEGF influenced the growth of HNSCC (11) and tongue squamous cell carcinoma, one of the most common malignancies in the oral cavity $(11,12)$. However, VEGF expression in Tca8113 cells was previously unknown. In this study, we found that the human tongue carcinoma cell line Tca8113 highly expressed VEGF at the mRNA and protein levels. Therefore, we established the biological basis for inhibiting or blocking the growth and development of tongue squamous cell carcinoma by interfering with VEGF expression.

When VEGF was downregulated in Tca8113 cells by siRNA produced from transfected plasmids, the proliferative ability was decreased and the proportion of cells in the G0 phase and apoptotic state were increased. These findings indicated that VEGF modulated the growth and apoptosis of human tongue squamous cell carcinoma and further validated VEGF as a rational therapeutic target.

In our study, the recombinant vector with green fluorescence protein (GFP) targeting VEGF (VEGFsiRNA) was transfected into the Tca8113 cells. Compared with chemically synthesized siRNA, the vector-produced siRNA persisted for a longer period of time in transfected cells (13). With the GFP reporter gene, we were able to detect the efficiency of transfection by fluorescence microscopy and revealed that VEGF gene expression in Tca8113 cells was significantly silenced. It has been shown that when the expression of VEGF is suppressed, the binding of VEGF with its receptor can be blocked, and the proliferation of tumor cells and endothelial cells decreased (14). Therefore, our results suggest that the decreased proliferation of Tca8113 cells may be attributed to the siRNA-mediated suppression of VEGF production.

Some of the most important strategies in the treatment of malignancies include the induction of cell apoptosis and the regulation of the cell cycle, as suggested by Sasano and Suzuki (15). In our study, after silencing the expression of
VEGF, the proportion of cells in the G0-G1 phase and the apoptotic cells increased, which is in agreement with the findings of Sasano and Suzuki (15). Therefore, VEGF is important in the proliferation of tumors, and inhibition of VEGF expression with siRNA is potentially useful in the treatment of tongue squamous cell carcinoma.

Tumor cells often evoke specific immune responses; however, they fail to eliminate all tumor cells. Our previous studies revealed that in OSCC, the majority of the DCs were immature DCs, while the total amount of DCs decreased in the neoplasm nest and peripheral blood of patients with OSCC (16). To study the effect of VEGF on the differentiation of immature DCs to mature DCs, we co-cultured immature DCs with Tca8113 cells or VEGF-downregulated Tca8113 cells in the normal medium containing GM-CSF, IL-4 and TNF- $\alpha$, which induced the differentiation of immature DCs into mature DCs. Our results revealed that the expression of HLA-DR, CD86, CD40, CD83, CD1a and CD14 on mature DCs following co-culture with Tca8113 cells was significantly decreased, while the downregulation of VEGF in Tca8113 cells reversed this effect. Therefore, we inferred that the high VEGF expressing Tca 8113 cells inhibit the immature DCs differentiating to mature DCs. This result is in agreement with the study by Hasebe et al, in which monocyte-derived DCs from cancer patients cultured with GM-CSF plus IL-4 exhibited lower levels of CD11c, CD40, CD86 and HLA-DR expression as compared with those derived from healthy volunteers (10).

In our study, we found that the expression of HLA-DR, CD86, CD40, CD83, CD1a and CD14 increased significantly in the immature DCs co-cultured with VEGF-downregulated Tca8113 cells. This result revealed that DC phenotypes were restored to a certain extent following the inhibition of VEGF. The functional activity of DCs depends, in part, on the expression of HLA-DR and co-stimulatory molecules (17). Reduced expression of these cell surface receptors, as observed in the presence of Tca8113 cells, may thus cause altered functionality of DCs. Alfaro et al found that DCs still matured under the 
effect of VEGF but they expressed less HLA-DR and CD86, and this effect was suspended by the VEGF inhibitor (18).

VEGF also suppressed the surface molecules of mature DCs. We found that the expression of HLA-DR, CD86, CD80, CD40 and CD14 on mature DCs decreased in the presence of Tca 8113 cells. However, when mature DCs were co-cultured with VEGF-downregulated Tca8113 cells, the expression of HLA-DR, CD86, CD80, CD40 and CD14 on the DCs was restored. This observation indicated that Tca8113 cells inhibited mature DCs from maintaining their mature status. Moreover, when we co-cultured DCs with VEGFdownregulated Tca8113 cells, the proportion of mature DCs increased to a certain extent. Therefore, siRNA targeting of the VEGF gene was capable of relieving the inhibition of VEGF on DC maturation and improving the function of DCs. Our results further support other previous findings indicating that an increased VEGF is correlated with the reduced number of DCs in tumor tissue and in the peripheral blood of patients with various types of cancer $(10,19)$. VEGF is known to promote tumor growth and inhibit the activation of nuclear factor $\kappa \mathrm{B}$ $(\mathrm{NF}-\kappa \mathrm{B})$ in endothelial progenitor cells, thereby inhibiting endothelial progenitor cells from differentiating into mature DCs (20). We speculated that VEGF released by Tca 8113 cells induce monocytes differentiating into endothelial cells but not mature DCs. The VEGF-induced endothelial cells may also be involved in angiogenesis in the cancer tissue.

In conclusion, siRNA targeting the VEGF gene is capable of inhibiting Tca8113 cell growth, inducing apoptosis and relieving the inhibition of VEGF on DC maturation. VEGF siRNA may be a novel and promising therapeutic strategy for the treatment of OSCC.

\section{Acknowledgements}

This study was supported by a grant from the National Natural Science Foundation of China (No. 81072213), Nanjing Medical Science and Research Project (No. YYK11039), the Jiangsu Health Science and Research Project(No.H200944), the Jiangsu Provincial Natural Science Foundation (No. BK2009043) and the Nanjing Science and Technology Development Plan (No. 201001084). The authors thank Shanghai Ninth Hospital for kindly providing the Tca8113 cell line.

\section{References}

1. Balkwill $\mathrm{F}$ and Mantovani A: Inflammation and cancer: back to Virchow? Lancet 357: 539-545, 2001.

2. O'Donnell RK, Kupferman M, Wei SJ, et al: Gene expression signature predicts lymphatic metastasis in squamous cell carcinoma of the oral cavity. Oncogene 24: 1244-1251, 2005.
3. Franovic A, Holterman CE, Payette J, et al: Human cancers converge at the HIF-2alpha oncogenic axis. Proc Natl Acad Sci USA 106: 21306-21311, 2009.

4. Wang ZY, Shi PH, Huang XF, et al: Peripheral blood dendritic cells and vascular endothelial growth factor in oral squamous cell carcinoma: correlation analysis and in vitro study. Int J Oral Maxillofac Surg 39: 713-720, 2010.

5. Bennaceur K, Chapman J, Brikci-Nigassa L, et al: Dendritic cells dysfunction in tumour environment. Cancer Lett 272: 186-196, 2008.

6. Kyzas PA, Cunha IW and Ioannidis JP: Prognostic significance of vascular endothelial growth factor immunohistochemical expression in head and neck squamous cell carcinoma: a metaanalysis. Clin Cancer Res 11: 1434-1440, 2005.

7. Gallucci S, Lolkema M and Matzinger P: Natural adjuvants: endogenous activators of dendritic cells. Nat Med 5: 1249-1255, 1999.

8. Fang L, Hu Q, Hua Z, Li S and Dong W: Growth inhibition of a tongue squamous cell carcinoma cell line (Tca8113) in vitro and in vivo via siRNA-mediated down-regulation of skp2. Int J Oral Maxillofac Surg 37: 847-852, 2008.

9. Wang Q, Huang Y, Ni Y, Wang H and Hou Y: siRNA targeting midkine inhibits gastric cancer cells growth and induces apoptosis involved caspase-3,8,9 activation and mitochondrial depolarization. J Biomed Sci 14: 783-795, 2007.

10. Hasebe H, Nagayama H, Sato K, et al: Dysfunctional regulation of the development of monocyte-derived dendritic cells in cancer patients. Biomed Pharmacother 54: 291-298, 2000

11. Ha PK and Califano JA: The molecular biology of mucosal field cancerization of the head and neck. Crit Rev Oral Biol Med 14. 363-369, 2003

12. Muller A, Sonkoly E, Eulert C, et al: Chemokine receptors in head and neck cancer: association with metastatic spread and regulation during chemotherapy. Int J Cancer 118: 2147-2157, 2006.

13. Brummelkamp TR, Bernards R and Agami R: A system for stable expression of short interfering RNAs in mammalian cells. Science 296: 550-553, 2002.

14. Bhat TA and Singh RP: Tumor angiogenesis-a potential target in cancer chemoprevention. Food Chem Toxicol 46: 1334-1345, 2008.

15. Sasano H and Suzuki T: Pathological evaluation of angiogenesis in human tumor. Biomed Pharmacother 59 (Suppl 2): S334-S336, 2005.

16. Shi PH, Hu QG, Wang ZY, et al: Research on the correlation of vascular endothelial growth factor and dendritic cell in patients with oral squamous cell carcinoma. Zhonghua Kou Qiang Yi Xue Za Zhi 44: 135-139, 2009.

17. Théry $\mathrm{C}$ and Amigorena S: The cell biology of antigen presentation in dendritic cells. Curr Opin Immunol 13: 45-51, 2001.

18. Alfaro C, Suarez N, Gonzalez A, et al: Influence of bevacizumab, sunitinib and sorafenib as single agents or in combination on the inhibitory effects of VEGF on human dendritic cell differentiation from monocytes. Br J Cancer 100: 1111-1119, 2009.

19. Lee BN, Follen M, Rodriquez G, et al: Deficiencies in myeloid antigen-presenting cells in women with cervical squamous intraepithelial lesions. Cancer 107: 999-1007, 2006.

20. Fricke I and Gabrilovich DI: Dendritic cells and tumor microenvironment: a dangerous liaison. Immunol Invest 35: 459-483, 2006. 